\title{
Recent advances in the laboratory detection of carbapenemase-producing Enterobacteriaceae
}

\author{
Yasufumi Matsumura ${ }^{\mathrm{a}, \mathrm{b}, \mathrm{c}, \mathrm{d}}$ and Johann DD Pitout ${ }^{\mathrm{b}, \mathrm{c}, \mathrm{d}, \mathrm{e}, \mathrm{f} *}$ \\ a Department of Clinical Laboratory Medicine, Kyoto University Graduate School of Medicine, Kyoto, Japan \\ ${ }^{\mathrm{b}}$ Departments of Pathology \& Laboratory Medicine, University of Calgary, Calgary, Canada \\ ${ }^{c}$ Microbiology, Immunology and Infectious Diseases, University of Calgary, Calgary, Canada \\ ${ }^{\mathrm{d}}$ Snyder Institute for Chronic Diseases, University of Calgary, Calgary, Canada \\ ${ }^{\mathrm{e}}$ Division of Microbiology, Calgary Laboratory Services, University of Calgary, Calgary, Canada \\ ${ }^{\mathrm{f}}$ Department of Medical Microbiology, University of Pretoria, Pretoria, South Africa \\ Corresponding Author*: \\ Johann DD Pitout, Calgary Laboratory Services, \#9, 3535 Research Road NW, Calgary, Alberta, CANADA, \\ T2L $2 \mathrm{~K} 8$
}




\section{Abstract}

Carbapenemase-producing Enterobacteriaceae (CPE), mainly Klebsiella pneumoniae and Escherichia coli, have been increasing rapidly on a global scale and are considered to be significant health threats. The most common carbapenemases are KPCs, NDMs, OXA-48-like, IMPs and VIMs but their distribution and prevalence differs between countries. The accurate, simple, cost effective and rapid detection of carbapenemases in clinical laboratories is an important initial step to control the spread of CPE within institutions. The diversity of carbapenemases in general, has challenged a simple approach for the detection of most types of CPE. This article summarizes the current and describes newer techniques available for the detection of carbapenemases among Enterobacteriaceae. We also provided a simplified approach for the accurate and rapid detection of CPEs that can easily be implemented in a clinical diagnostic laboratory.

Keywords: Carbapenemase-producing Enterobacteriaceae, Laboratory detection. 


\section{Introduction}

The Enterobacteriaceae, most notably Escherichia coli, and Klebsiella pneumoniae, are among the most important causes of serious hospital-acquired and community-onset bacterial infections in humans [1]. The global spread of antimicrobial resistance was recently identified by the World Health Organization (WHO) as one of the three greatest threats to human health and is a public health threat [2]. Moreover, the WHO released a report in 2014 entitled: "Antimicrobial resistance: global report on surveillance" that focused on antibiotic resistance in seven different bacteria responsible for common, serious diseases such as bloodstream infections, diarrhea, pneumonia, urinary tract infections and gonorrhoea [3]. It states that antimicrobial resistance to common bacteria has reached alarming levels in many parts of the world and that in some settings, few, if any, of the available treatments options remain effective for common infections. [3].

The spread of multi-resistant bacteria is problematic for the medical community at large since it undermines empirical treatment regimens by delaying the administration of appropriate antibiotic therapy and by reducing the options for appropriate treatment. This contributes to increased patient mortality and morbidity [4]. The problem is so serious that it threatens the achievements of modern medicine.

One of the most urgent areas of antimicrobial drug resistance is the rapid evolution of carbapenem resistance in Enterobacteriaceae which has spread globally and rapidly during the last 
decade [5]. Carbapenems are often the last line of effective therapy available for the treatment of serious infections due to multidrug resistant bacteria. Resistance to carbapenems involves multiple mechanisms, including alterations in outer membrane permeability mediated by the loss of porins, upregulation of efflux systems combined with high levels of AmpC cephalosporinases or other $\beta$-lactamases [6]. Enzymes that hydrolyze the carbapenems, referred to as carbapenemases, are the most important causes of carbapenem resistance among Gram negative bacteria [6].

The clinical diagnostic laboratory often acts as an early warning system, alerting the medical community to new resistance mechanisms present in clinically important bacteria. The presence of carbapenemases among Enterobacteriaceae in hospitalised patients, is considered by most infection control practitioners as infection control emergencies [7]. Some clinical laboratories may not be fully aware of the importance and the methods for detecting bacteria with carbapenemases [8]. The consequences have been several treatment failures in patients who received inappropriate antibiotics and outbreaks of multidrug-resistant gram-negatives which required expensive control efforts. A recent example occurred in Alberta, Canada when the nosocomial outbreak of carbapenemase-producing Enterobacteriaceae and Acinetobacter baumannii resulted in death of a patient that was attributed to a different patient with recent foreign hospitalization [9].

Clinical microbiology laboratories should be able to rapidly detect carbapenemase-producing Enterobacteriaceae (CPE). This article will address the laboratory 
detection of carbapenemases among isolates and provide a simplified approach for the accurate and rapid detection of CPEs that can easily be implemented in a clinical diagnostic laboratory. The detection of CPEs directly on patient specimens (e.g. the use of CHROMagar media etc.) is outside the scope of this article.

\section{Carbapenemases}

Carbapenemases belong to the molecular class A (i.e. KPC types), the class B, (or the metallo- $\beta$-lactamases) [i.e. VIM, IMP and NDM types] and the class D oxacilinases (i.e. OXA-48-like enzymes). The NDM, OXA-48-like, KPC, IMP and VIM types are the most common global carbapenemases among CPE [7]. Other types of carbapenemases (e.g. GES, SME, IMI, and NMC) that are far less commonly encountered in the clinical laboratory and will not be addressed in this article.

The class A KPC-type B-lactamases have been extensively and almost exclusively reported in K. pneumoniae [10]. To date more than 20 different KPC variants have been described even though KPC-2 and -3 remains the most dominant variants [11]. These enzymes provide resistance to the penicillins, carbapenems, cephalosporins, cephamycins and monobactams and are inhibited by $\beta$-lactamase inhibitors such as clavulanic acid (weakly), tazobactam (weakly), boronic acid and avibactam. KPC $\beta$-lactamases (especially KPC-2 and -3) have been described in several enterobacterial species, especially Klebsiella spp. and to a lesser extend in Enterobacter spp and E. 
coli. [12]. Several nosocomial outbreaks most often due to K. pneumoniae, have been reported from North America (especially the USA), South America (Colombia, Argentina), Europe (Greece, Italy, Poland), Asia (China) and Middle East (Israel) $[11,13,14]$. KPC-producing bacteria are endemic in these regions [14]. K. pneumoniae ST258 with KPC-2 and KPC-3 had significantly contributed to the world-wide distribution of this resistance trait [14].

The class B ß-lactamases or metallo- $ß$-lactamases (MBLs) had been identified in various enterobacterial species including K. pneumoniae, E. coli and Enterobacter spp. [7]. They mainly consist of NDM-, VIM-, and IMP-type enzymes, with the first type are endemic in certain regions such as South Asia. Although IMP producers are mainly identified in China, Japan, and Australia, VIM-producing K. pneumoniae isolates are mainly found in Italy and Greece [7]. Since the first description of NDM-1, more than 10 variants of this enzyme has been described, the majority of them originated from Asia [8]. The majority of NDM-producing bacteria are broadly resistant to various drug classes and also carry a diversity of additional resistance mechanisms [7]. These include plasmid-mediated AmpC $\beta$-lactamases (especially CMY types), ESBLs (especially CTX-M-15), different carbapenemases (e.g. OXA-48-, VIM-, KPC-types), 16S ribosomal RNA methyltransferases, plasmid-mediated quinolone resistance determinants, macrolide modifying esterases, and rifampicin-modifying enzymes. Consequently, Enterobacteriaceae with NDMs remain only susceptible to agents such colistin, fosfomycin and tigecycline [8]. 
The class D carbapenem-hydrolyzing $ß$-lactamase found in $K$. pneumoniae isolates is OXA-48 (and derivatives) that was firstly reported from a K. pneumoniae isolate from Turkey [15]. OXA-48 hydrolyses efficiently narrow-spectrum ß-lactams such as penicillins, weakly hydrolyses carbapenems, and spares broad-spectrum cephalosporins [16]. It has been found among all Enterobacteriaceae however it is mostly identified in $K$. pneumoniae (mostly from nosocomial origin) and E. coli (mostly from community origin) isolates. OXA-48-producing K. pneumoniae is endemic in Turkey and certain North African countries (e.g. Morocco, Tunisia) showing a wide range of susceptibility profiles [15]. Indeed MICs of carbapenems may significantly vary from isolate to isolate, depending on the host permeability background. Similarly, susceptibilities to broad-spectrum cephalosporins can also significantly vary, depending on the co-production of other B-lactamases such as the ESBLs. Some OXA-48 derivatives have also been identified in K. pneumoniae, being OXA-181, OXA-204, and OXA-232, all sharing similar hydrolytic properties [17]. These enzymes have been identified in North Africa, Australia, New Zealand, but one of the main sources of OXA-181 (which is the second most common OXA-48 derivative) is the Indian subcontinent.

K. pneumoniae has been the most common species among CPE, followed by E. coli but carbapenemase producers has been found in various other Enterobacteriacae, such as Enterobacter spp., Citrobacter spp., Serratia spp, and Proteus spp. A summary of the characteristics and distribution of the five major carbapenemases are shown in Table 1. It is important to remember that 
Table 1. Characteristics of carbapenemases among Enterobacteriaceae.

\begin{tabular}{|c|c|c|c|c|}
\hline Amber class & Enzyme & $\begin{array}{l}\text { Prevalent } \\
\text { subtype }\end{array}$ & Spectrum & Endemic area \\
\hline A & KPC & KPC-2, KPC-3 & $\begin{array}{c}\text { Penicillins, cephalosporins, } \\
\text { cephamycins, aztreonam, } \\
\text { carbapenems }\end{array}$ & $\begin{array}{c}\text { United States, Greece, Italy, } \\
\text { Poland, Israel, Brazil, Colombia, } \\
\text { Argentina, China, Taiwan }\end{array}$ \\
\hline B & NDM & NDM-1 & $\begin{array}{l}\text { Penicillins, cephalosporins, } \\
\text { cephamycins, carbapenems }\end{array}$ & $\begin{array}{l}\text { India, Pakistan, Sri Lanka, United } \\
\text { Kingdom, France, Balkan states, } \\
\text { Arabian peninsula, North African } \\
\text { countries }\end{array}$ \\
\hline B & IMP & $\begin{array}{l}\text { IMP-1-like, } \\
\text { IMP-2-like }\end{array}$ & $\begin{array}{l}\text { Penicillins, cephalosporins, } \\
\text { cephamycins, carbapenems }\end{array}$ & Japan, Taiwan, China \\
\hline B & VIM & VIM-1-like & $\begin{array}{l}\text { Penicillins, cephalosporins, } \\
\text { cephamycins, carbapenems }\end{array}$ & $\begin{array}{c}\text { Greece, Spain, Italy, South Korea, } \\
\text { Taiwan }\end{array}$ \\
\hline
\end{tabular}


others

(weak) 
carbapenemases have different features which will affect the diagnostic performance of laboratory tests designed to detect CPE [8].

\section{Detection of Enterobacteriaceae with carbapenemases}

The detection of carbapenemases in Enterobacteriaceae consists of a two-step approach namely a screening process using the carbapenems followed by a confirmation test to detect the presence of a carbapenemase in isolates that testes non-susceptible to the carbapenems. As mentioned before, Enterobacteriaceae with OXA-48-like enzymes can test susceptible to the broad-spectrum cephalosporins (e.g. $3^{\text {rd }}$ and $4^{\text {th }}$ generation cephalosporins) and the carbapenems and the laboratory detection of such bacteria remains a challenge to most clinical laboratories.

\section{Screening for non-susceptibility (NS) to the carbapenems}

$\mathrm{CPE}$ are often NS to carbapenems and this remains a simple initial screen for the presence of carbapenemases among Enterobacteriaceae. However, the choice which carbapenems to use for screening with subsequent break points remains a controversial issue. Some CPEs, especially the OXA-48 producers, show only slight increases in carbapenem MICs [18,19]. For the detection of OXA-48-like producing isolates, a recent Belgium study advocates the use of NS to piperacillin-tazobactam and temocillin. This combination shows the best sensitivity (i.e. up to 98\%) for K. pneumoniae but low specificity for the detection of OXA-48-like producers in especially in non- K. pneumoniae Enterobacteriaceae [19]. 
The Clinical and Laboratory Standards Institute (CLSI) in 2010 revised their breakpoints for the carbapenems and do not recommend routine confirmation tests for carbapenemases if the current interpretive criteria are used. CLSI do advocate the detection of carbapenemases in Enterobacteriaceae for infection control purposes [20]. The European Committee on Antimicrobial Susceptibility Testing (EUCAST) uses different carbapenem screening breakpoints from clinical breakpoints [21]. In the EUCAST guidelines, the meropenem MIC of $>0.12 \mu \mathrm{g} / \mathrm{ml}$ or disk diameter $\leq 25 \mathrm{~mm}$ are recommended for the screening of carbapenemases in Enterobacteriaceae. The EUCAST meropenem screening criteria have difficulty to detect some OXA-48-producers [19]. Only $80 \%$ of CPE clinical isolates from Belgium and France were detected using the EUCAST meropenem criteria; $25 \%$ of OXA-48-producers and 19\% of VIM-1-producers tested susceptible to meropenem.

Ertapenem non-susceptibility has excellent sensitivity but poor specificity for CPE, especially in species such as Enterobacter spp. due to the presence of high-level production of AmpC $\beta$-lactamases in combination with porin loss [8]. Imipenem MICs breakpoints cannot reliably separate wild-type isolates from carbapenemase producers in species such as Proteus spp., Providencia spp., and Morganella morganii. Faropenem is an oral penem antibiotic and one UK study evaluated its use as a potential screening agent for CPE [22]. Using clinical isolates with several types of carbapenemases, including OXA-48 producers, growth up to the edge of a $10 \mu \mathrm{g}$ 
faropenem disc showed 99\% sensitivity and 94\% specificity for the detection of CPE [22].

The most current approach to screen for CPE should at least include ertapenem in combination with imipenem or meropenem [23]. The combination of piperacillin-tazobactam in temocillin is an option to screen for OXA-48-like producers [19]. Faropenem is an attractive option but additional evaluation of this agent is needed.

\section{Confirmation tests: Phenotypic methods}

Phenotypic methods that detect the enzyme activity of carbapenemases will be able (in theory at least) to identify all types of carbapenemases, including novel enzymes.

\section{a. Inhibitor-based synergy tests}

Inhibitor-based synergy tests are based on the ability of certain substrates to inhibit the action of carbapenemases [24]. This process involves the testing of a carbapenem with and without the addition of an inhibitor that is specific to the type of carbapenemase. The inhibitor-based methods are often based on disk susceptibility testing such as the double-disk synergy test (DDST) and combined disk test (CDT). In the DDST, an inhibitor disk is placed near a carbapenem disk and presence of carbapenemase expands the growth-inhibitory zone between the two disks. This is often referred to the "keyhole" or "champagne cork" sign. In the CDT, a carbapenem disk with and without an inhibitor are used and an increase in inhibitory zone diameters indicates the presence of carbapenemase. The interpretation tends to be subjective with the DDST but is standardized with the 
CDT (e.g. zone diameter difference of Xmm is indicative of a positive test).

Boronic acid derivatives (phenylboronic acid [PBA] and 3-aminophenylboronic acid [ABPA]) are often used for the inhibition of KPCs [24]. These agents have a broad-spectrum of inhibition and also inhibit other $\beta$-lactamases such as AmpC $\beta$-lactamases. Cloxacillin, an AmpC inhibitor without activity against KPC, can be used to discriminate between KPCs and AmpCs. Inhibitors for MBLs (i.e. NDM, IMP, and VIM) include metal chelators such as EDTA, dipicolinic acid (DPA), 2-mercaptopropionic acid, and sodium mercaptoacetic acid [24]. Chemical compounds with specific inhibitor properties for OXA-48-like enzymes are not been yet available for the identification of these carbapenemases.

For the reliable detection of the most common carbapenemases among CPE using an inhibitor-based approach, clinical laboratories should use a combination of inhibitors for the detection of KPC (i.e. boronic acid) and MBLs (i.e. metal chelator). Other supplementary tests (i.e. NS to piperacillin-tazobactam and temocillin) are necessary to screen for the presence of OXA-48-like producers [19]. Several investigators have evaluated synergy testing using boronic acid (for KPCs), boronic acid with cloxacillin (to distingisuh between KPCs and AmpCs), and DPA or EDTA (for MBLs) [25-27]. Commercial disk kits have been available and include the KPC/MBL Confirm Kit (Rosco Diagnostica, Taastrup, Denmark) and the Mastdiscs ${ }^{\mathrm{TM}}$ Carbapenemase Detection Set (MAST GROUP, Merseyside, UK). Both assays were evaluated and showed $>97 \%$ sensitivity 
and 93\% specificity for KPC and NDM-producers [28]. However, about a half of IMP and VIM producers were not detected. Giske et al described an inhibitor-based method that performed better with IMP and VIM CPE [26]. The overall sensitivities for detection of all clinical CPEs isolates remained $78 \%-80 \%$ due to inability for the commercial tests to detect OXA-48-like producers. Recently, Rosco Diagnostica and Liofilchem (Roseto degli Abruzzi, Italy) launched new disk kits which includes the addition of temocillin for the screening of OXA-48-like producers (KPC/MBL \& OXA-48 Confirm kit, Rosco Diagnostica, and KPC\&MBL\&OXA-48 disks kit, Liofilchem $\left.{ }^{\circledR}\right)$. These approaches have not yet officially been evaluated.

Miriagou et al. used two types of inhibitors (i.e. DPA and PBA) in combination with meropenem for the identification of CPE that simultaneously produce KPCs and VIMs [29]. Several other investigators used similar approaches to accurately detect CPE that produce both class A and class B carbapenemases [30,31]. Maurer et al. recently published an extensive algorithm that include synergy tests using cloxacillin supplemented agar with ABPA, EDTA, and temocillin disks which resulted in $100 \%$ sensitivity and specificity for detecting CPE that contain the most common types of carbapenemases [32]. Tsakris et al. described an OXA-48 disk confirmation test that used an imipenem disk impregnated with EDTA and EDTA + PBA [33]. The interpretation of the test is based on the distortion of zone sizes and is extremely subjective and very difficult to interpret.

Inhibitor-based disk tests are very popular with certain clinical laboratories due to their user 
friendliness, cost-effectives and the availably of commercial tests. These approaches should at least include 2 types of inhibitors (e.g. boronic acid derivatives for KPCs and metal chelators for MBLs) and are especially reliable for the detection of KPCs and NDMs. The prolonged turn-around times and lack of inhibitors specific for OXA-48-like carbapenemases curtail the use of inhibitors as a stand-alone approach for the detection of CPE.

\section{b. Modified Hodge test (MHT)}

The modified Hodge test (MHT) or clover leaf technique is a phenotypic confirmation test for carbapenemases that is currently proposed by the CLSI) [20]. This test is based on the inactivation of a carbapenem by CPEs that enable a carbapenem-susceptible indicator strain to extend growth toward a carbapenem-containing disk, along the streak of inoculum of the tested isolate. The MHT is cost-effective, easy to perform but unfortunately difficult to interpret in some instances and false-positive results are a concern especially among non-K. pneumoniae [34-36]. Moreover, the turn-around time is around 16-18 hours. The MHT has an excellent sensitivity for detecting CPE with $b l a_{\mathrm{KPCs}}$ and $b l a_{\mathrm{OXA}-48-\mathrm{like}}$ but performs poorly in detecting those with $b l a_{\mathrm{NDMs}}$ $[28,35]$. However it remains a valuable option in clinical laboratories with for the detection of CPE especially in KPC and OXA-48-like endemic regions.

\section{c. Carbapenem Inactivation Method}

Carbapenem Inactivation Method (CIM) is a new method described in 2015 [37]. This 
method involves a suspension of the test isolate in $400 \mu$ water to which a meropenem disk is added and incubated at $35^{\circ} \mathrm{C}$ for approximately two hours. The meropenem disk is then placed on an agar plate inoculated with a susceptible $E$. coli indicator strain and subsequently incubated at $35^{\circ} \mathrm{C}$ for another $12-18$ hours. If the test isolate produced a carbapenemase, the meropenem will be inactivated allowing uninhibited growth of the susceptible indicator strain (i.e. no zone of inhibition). Isolates without carbapenemases showed inhibition zones. The test can be read after 6 hours but the best results are obtained after overnight incubation. This method showed high concordance with results obtained by PCR to detect genes coding for the following carbapenemases: KPC, NDM, OXA-48, VIM, IMP and OXA-23 [37].

A different group evaluated the CIM and found it be $98.8 \%$ sensitive and $100 \%$ specific to detect CPE with OXA-48-like, NDM and KPC that tested previously negative with the Carba NP test [38]. One IMP-producing mucoid isolate was negative for both the CIM and the Carba NP test. The advantages of CIM included cost-effectives, reagents that are readily available in most clinical laboratories and it is easy to perform. Unfortunately this test is time consuming since the best results are obtained when agar plates are incubated for 12-18 hours.

\section{d. The Carba-NP test}

The Carba-NP test was developed by Nordmann and Poirel in 2012 and the CLSI recommends its use as a confirmation test since 2015 [39]. This method involves the incubation of a 
test bacterium (the lysate) with a solution containing imipenem, zinc sulfate, and phenol red. The production of carbapenemases is indicated detected by $\mathrm{pH}$ change due to the hydrolysis of imipenem (i.e. a color change from red to orange or yellow). The initial study from Nordmann and Poirel reported $100 \%$ sensitivity and $100 \%$ specificity for CPE with bla $a_{\mathrm{KPC}}$, bla $a_{\mathrm{NDMs}}$ bla $a_{\mathrm{IMPs}}$, bla $a_{\mathrm{VIMs}}$ and

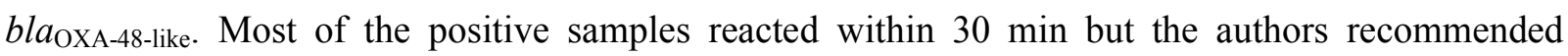
incubation for up to $2 \mathrm{~h}$. The Carba-NP test has been validated with colonies grown on Mueller-Hinton, blood, and trypticase soy agar plates but it cannot be performed with colonies obtained from Drigalski or McConkey agar plates.

During various validation and verification studies, some detection issues with the carba NP test have been found and improved on to some extent. Mucoid K. pneumoniae and some CPE with with OXA-48-like often give false negative results and remain problematic for the Carba-NP test. Tijet et al. reported a sensitivity of $21 \%$ for OXA-48-like producers and poor results were obtained especially with mucoid $K$. pneumoniae isolates [40]. They reported that increasing the bacterial inoculum improved the sensitivity of the Carba-NP test for OXA-48-like producers to 59\%. The Carba-NP methodology was recently standardized by the CLSI and incorporated into the 2015 CLSI guidelines [20]. That guideline shows a detailed protocol with appropriate control reactions and isolates. This CLSI standardized method demonstrated an excellent sensitivity and specificity, and positive results were obtained within $15 \mathrm{~min}$ in $94 \%$ of CPE with various carbapenemases [41]. One 
of the major drawbacks of the Carba-NP test, is that laboratories need to prepare most of reagents. The cost of imipenem powder can be as high as $\$ 317$ for $100 \mathrm{mg}$. At least $10 \mathrm{mg}$ is needed to make solution which can be used for 13 samples and stored for up to 3 days. However, a cheaper alternative, intravenous imipenem/cilastatin powder (approximately $\$ 4$ per $100 \mathrm{mg}$ of imipenem) provided similar performance than pure imipenem powder [42]. Commercial versions have recently been launched for clinical use and include the RAPIDEC $®$ CARBA NP (bioMérieux), Rapid CARB Screen (Rosco Diagnostica), and Neo-Rapid CARB (updated version of Rapid CARB Screen; Rosco Diagnostica) kits. These kits are convenient and RAPIDEC ${ }^{\circledR}$ CARBA NP and Neo-Rapid CARB had similar performances to the manual method $[42,43]$.

Additional developments or applications of the carbaNP tests have recently been reported. A modification called the Carba NP test II has the ability to identify which type of carbapenemase is present among $\mathrm{CPE}$. The original version is combined with $\beta$-lactamase inhibitors and this medication has the ability to distinguish between class A, B, and D $\beta$-lactamases [44]. The presence of class D $\beta$-lactamases is deduced from a lack of inhibition by both class A and B inhibitors. A shorter turn-around time of the original carba NP test was reported by Lee et al. when they performed this method on five hour old bacterial cultures [45]. Nordmann and Poirel also described the direct detection of $\mathrm{CPE}$ on blood culture bottles that flagged positive and were Gram negative on stains [46]. This assay showed a 100\% sensitivity and specificity for all types of CPE, excluding for 
OXA-48-like producers (91.3\% sensitivity and 100\% specificity). This method enables the rapid reporting of carbapenemases from blood cultures up to 24 hours earlier than from conventional growth on agar plates

The Carba NP test and its modifications are relatively easy to perform and provide rapid results, especially for CPE with $b l a_{\mathrm{KPC}}$, and $b l a_{\mathrm{NDM}}$. However, this test can be challenging for some technologists to interpret (due to various ranges of the orange colour) and OXA-48-like producers remain a problem. Moreover, the commercial versions are expensive and showed a sensitivity of $98 \%$ and specificity of $99 \%$ for detecting different types of CPE [47].

\section{e. The Blue Carba test}

The Blue-Carba test is another modification of the Carba-NP test developed in Portugal by Peixe and colleagues. This method uses bromothymol blue as the indicator, imipenem/cilastatin as the antibiotic/substrate and can be performed directly on bacterial colonies (as opposed to bacterial extracts in the original Carba NP test) [48]. The Blue-Carba test performs very well for KPC and MBL CPE with low MICs to the imipenem but gives similar results and has the same issues as the CarbaNP test regarding OXA-48-like CPE [49-51]. The commercial version has been recently launched as the Rapid Carb Blue kit (Rosco Diagnostica) [52].

\section{f. Starch-iodine assay}

The starch-iodine assay is based on color changes of a starch-iodine compound (i.e. from 
dark pink to clear) due to the release of hydrogen ions that occurs during the hydrolysis of the $\beta$-lactam ring. A commercial version, the Carbapenembac assay, comprised of a strip that contains imipenem and starch [53]. A bacterial suspension is deposited onto the strip and incubated for 10 min, followed by the addition of iodine. A change in color from dark pink to clear within 30 min is indicative for the presence of carbapenemases. CPE with bla $a_{\mathrm{KPCs}}$ were detected with $100 \%$ sensitivity and specificity. The Carbapenembac assay is cost effective and easy to perform but further validation using CPEs with different carbapenemases need to be performed.

\section{g. Immunochromatography}

Immunochromatography is based on an antigen-antibody reaction performed on chromatographic paper. A commercial version named Quick Chaser® IMP (Mizuho Medy, Saga, Japan) was designated to detect all IMP-type carbapenemases. This test detects the presence of IMPs in Enterobacteriaceae and non-glucose-fermenting gram-negative rods with $100 \%$ sensitivity and $100 \%$ specificity [54]. Results are obtained within $15 \mathrm{~min}$ after three drops of bacterial colonies suspended in an extract solution are applied to a test cartridge. The assay is easy to perform and provides rapid results but it not relevant in regions non-endemic for CPE with $b l a_{\text {IMPs. }}$ Glupczynski et al. recently evaluated 2 new commercial immunochromatograhic assays (OXA-48 K-SeT® and KPC K-SeT®, Coris Bioconcept, Gembloux, Belgium) that showed 100\% sensitivity and specificity for the detection of OXA-48-like and KPC CPE respectively [55]. These tests were easy to perform with 
very short turn-around time (15 $\mathrm{min})$.

\section{f. Electrochemical Assay}

Another innovative method is the electrochemical detection of carbapenem hydrolysis (BYG Carba test from Belgium) whicthe change of $\mathrm{pH}$ and redox activity following imipenem hydrolysis and that detects the presence of carbapenemase within 30 minutes [56]. The BYG Carba tests in comparison with PCR results, displayed 95\% sensitivity and 100\% specificity. This technique is novel, rapid and efficient based on an electro-active polymer biosensing technology discriminating between $\mathrm{CPE}$ and non-CPE. The precise electrochemical signal (i.e. electrochemical impedance variations) allowed for real time objective measurement and interpretation criteria which should facilitate the accreditation process of this technology.

\section{g. Spectrophotometry}

A clinical application for using ultraviolet spectrophotometry to determine imipenem hydrolysis was developed in 2012 and has the ability to detect the most clinically relevant CPEs with $100 \%$ sensitivity and $98.5 \%$ specificity [57]. Bacterial proteins are extracted after sonication and mixed with imipenem and buffer. UV absorbance was recorded at $297 \mathrm{~nm}$ for $10 \mathrm{~min}$ and slope per minute was used to distinguish hydrolysis from the self-degradation of imipenem. A follow-up study [58] report a sensitivity of $100 \%$ and specificity of $76.7 \%$ [58]. The UV spectrophotometry assay is labor intensive and not routinely available in most diagnostic clinical laboratories. 


\section{h. Mass spectrometry}

Matrix-assisted laser desorption ionization-time of flight mass spectrometry (MALDI-TOF)

has been recently introduced into clinical microbiology laboratories for identification of bacterial species. Two MALDI-TOF MS systems (Microflex LT [Bruker Daltonics, Germany] and VITEK MS [bioMérieux, France]) are in clinical use and provide a rapid, inexpensive (after the initial financial outlay), and accurate identification of most bacterial species. The Bruker system includes software for analysis of raw spectra data (i.e. FlexAnalysis) and has been used to detect the presence of carbapenemases.

Carbapenemase-hydrolyzed degradation products were measured by MALDI-TOF after the 2-3 hour incubation of bacterial cultures with carbapenems (i.e. meropenem or ertapenem) solutions $[59,60]$. Excellent sensitivities and specificities of MALDI-TOF were reported. Unfortunately, false-negatives were reported mostly due to the weak carbapenemase activities of OXA-48-like producers as well as the interactions of polysaccharides present in highly mucoid isolates [61]. Recently Sauget et al. reported a sensitivity of $98.9 \%$ and a specificity of $97.8 \%$ for the detection of OXA-48-producers using imipenem (as opposed to meropenem or ertapenem) [62]. Studentova et al. reported modified MALDI-TOF method which adds ammonium bicarbonate to the solution that enhances activity of OXA enzymes [63]. Wang et al. successfully used an automated statistical peak analysis software program called ClinProTools in the Bruker system to detect carbapenem 
degradation products that negated the need of manual inspection of raw spectra. [64]. The MBT STAR-BL prototype software developed by Bruker also succeeded in the automated detect of carbapenem degradation products [65].

Another approach is to use MALDI-TOF for the detection of antimicrobial resistance determinants associated with carbapenemases. An example is the detection of pKpQIL_p019 protein present in some $b l a_{\mathrm{KPC}}$ containing plasmids [66]. The follow-up validation study using FlexAnalysis showed a $96 \%$ sensitivity and a 99\% specificity for the presence of KPC-producing CPE [67]. However, this approach is really only valid for molecular epidemiology studies.

The more advanced and expensive mass spectrometry systems, such as liquid chromatography-MS (LC-MS), tandem-MS (MS-MS), capillary electrophoresis-electrospray ionization-tandem-MS (CE-ESI-MS), and PCR-electrospray ionization-MS (PCR-ESI-MS) have also been evaluated to detect carbapenemases. However, these systems are utilized within the research setting and are rarely available in clinical laboratories.

The use of MALDI-TOF for the detection of CPE is currently in investigative stages and is not available for routine use. One of the drawback is the need of time-consuming preparation of reagents. However, this technology has the potential to rapidly and cost-effectively detect CPE. The initial financial outlay for acquiring MALDI-TOFF will curb the wide-spread use of this technology, especially in developing countries. 


\section{Molecular methods}

Molecular methods are excellent options for the confirmation of carbapenemases among Enterobacteriaceae. These tests are highly sensitive and specific for detecting different carbapenemase genes and are considered by some microbiologists to be the gold standard. Some assays have been validated for the identification of carbapenemase genes directly on clinical samples. Several in-house multiplex PCR's, commercial real time PCR and DNA microarray methods are available for routine use and should at least include the most clinically relevant carbapanemase genes (i.e. $b l a_{\mathrm{KPC}}, b l a_{\mathrm{NDM}}, b l a_{\mathrm{OXA}-48-\mathrm{like}}, b l a_{\mathrm{IMP}}$ and $\left.b l a_{\mathrm{VIM}}\right)$. Disadvantage of molecular methods includes the relative high costs (as compared to most phenotypic tests), the ability to only detect carbapenemase genes included in the assay (as opposed to hydrolysis assays) and longer turnaround times (as opposed to the rapid phenotypic tests). To negate the cost, some clinical laboratories will run the test in batches that can lead to delayed turnaround times. The use of automated commercial systems is easier to incorporate into clinical laboratories and may help to reduce the work load, turn-around-time and errors but such tests are often more expensive than in-house methods.

It should be noted that molecular assays will not be able to discover novel carbapenemase genes and some methods fail to detect all the variants. It is important to keep this in mind when deciding on implementing molecular tests for routine diagnostic testing.

\section{a. PCR-based methods}


The strategy for the identification of carbapenemase gene is to first amplify the specific gene (e.g. $b l a_{\mathrm{NDM}}$ ) using simplex PCR and then to sequence the amplicon for the identification of the subtype (e.g. bla $a_{\mathrm{NDM}-1}$ etc.). For the screening and detection of carbapenemases among clinical isolates, multiplex PCR assays that detect different types of genes (e.g. bla $a_{\mathrm{NDM}}, b l a_{\mathrm{KPC}}, b l a_{\mathrm{IMP}}$ etc) at the same time are useful and commonly used. A conventional in-house PCR method includes DNA extraction, PCR amplification, and gel electrophoresis steps. Table 2 shows an up-to-date list of in-house and commercial multiplex PCR assays currently available for clinical utilization. The most extensive multiplex assay available has the ability to identify 11 different carbapenemase genes in three separate reactions [68]. However, for the routine use in clinical laboratories, one-reaction multiplex PCR assay that includes the most clinical relevant genes (i.e. bla $a_{\mathrm{KPC}}, b l a_{\mathrm{NDM}}, b_{\mathrm{OXA}-48-\mathrm{like}}$, $b l a_{\mathrm{IMP}}$ and $b l a_{\mathrm{VIM}}$ are most often implemented [28].

The reagents and instruments used in conventional in house methods are cost-effective but time consuming (i.e. it takes 2 to 6 hours to get results) and relatively laborious. Real-time PCR method is superior to conventional method in terms of specificity, speed, and less labor intensive but is more expensive. Real time PCR uses melting curve analysis to confirm specific melting temperature (Tm) value of the target amplicon, or uses specific oligonucleotide probes to the target amplicon that detects an amplification signal. The probe assays are more specific than the melting curve assays. The amplification is monitored in a real-time manner negating the need for gel 
Table 2. PCR-based methods for the detection of carbapenemase genes.

\begin{tabular}{|c|c|c|c|c|c|c|c|c|}
\hline \multirow[b]{2}{*}{ PCR (details) } & \multirow[b]{2}{*}{ Assay name } & \multicolumn{3}{|c|}{ Target carbapenemase genes } & \multirow[b]{2}{*}{ Other genes } & \multirow{2}{*}{$\begin{array}{l}\text { No. } \\
\text { of } \\
\text { reacti } \\
\text { ons }\end{array}$} & \multirow[b]{2}{*}{ Comment } & \multirow[b]{2}{*}{$\begin{array}{l}\text { Refere } \\
\text { nce }\end{array}$} \\
\hline & & $\begin{array}{l}\text { KPC, NDM, OXA- } \\
48 \text {-like }\end{array}$ & $\begin{array}{l}\text { IMP, } \\
\text { VIM }\end{array}$ & Others & & & & \\
\hline Conventional & In-house & All & Both & $\begin{array}{l}\text { SPM, BIC, } \\
\text { AIM, GIM, } \\
\text { SIM, DIM }\end{array}$ & - & 3 & & {$[1]$} \\
\hline Conventional & In-house & All & Both & - & - & 1 & & {$[2]$} \\
\hline $\begin{array}{l}\text { Real-time } \\
\text { (evagreen, } \\
\text { melting analysis) }\end{array}$ & In-house & All & Both & GES & - & 1 & & [3] \\
\hline $\begin{array}{l}\text { Real-time (SYBR } \\
\text { green, melting } \\
\text { analysis) }\end{array}$ & In-house & All & Both & $\begin{array}{l}\text { GES, OXA- } \\
23\end{array}$ & - & 2 & $\begin{array}{l}\text { Tested on BD MAX } \\
\text { system. VIM-1 and VIM- } \\
2 \text { groups can be } \\
\text { discriminated. }\end{array}$ & {$[4]$} \\
\hline $\begin{array}{l}\text { Real-time } \\
\text { (molecular } \\
\text { beacon probe) }\end{array}$ & $\begin{array}{l}\text { Check-Direct CPE } \\
\text { (Checkpoints, } \\
\text { Wageningen, } \\
\text { Netherlands) }\end{array}$ & All & VIM & - & - & 1 & $\begin{array}{l}\text { NDM/VIM signal cannot } \\
\text { be differentiated (BD } \\
\text { MAX can) }\end{array}$ & {$[5]$} \\
\hline
\end{tabular}


electrophoresis detection step. Monteiro et al. developed a one-reaction assay for bla $a_{\mathrm{KPC}}, b_{\mathrm{NDM}}$, bla $a_{\mathrm{OXA}-48-\mathrm{like}}, b l a_{\mathrm{IMP}}, b l a_{\mathrm{VIM}}$ and $b l a_{\mathrm{GES}}$ using melting curve analysis [69]. A probe-based assay was developed by Lee et al. [70] but it targets only three carbapenemase genes. Increasing numbers of commercial real-time probe-based PCR assays are available for clinical laboratories. These include Check-Direct CPE (Checkpoints, Netherlands) [71].

To further reduce turn-around-time and decrease labor, fully automated real-time PCR systems that incorporate the DNA extraction step. Recently released tests available for clinical laboratory use included Unyvero, Eplex (GenMark), and PCR-ESI MS (IRIDICA, Abott).

\section{b. Non-PCR methods}

A non-PCR molecular rapid commercial confirmation test (LAMP; Easyplex superbug CRE from Amplex Diagnostics [Bahnhof, Germany]), uses loop-mediated isothermal amplification, for the detection of CPE (KPC, NDM, VIM, OXA-48-like) from a plate or directly on positive blood cultures [72]. The system gives results within 15 minutes.

\section{b. Microarrays}

The advantage of microarray-based assays is the ability to simultaneously detection a large number of carbapenemase genes. An example of a commercial microarray is the Check-MDR CT103 (Checkpoints, Wageningen, Netherlands) assay that has the ability to detect 11 different $\beta$-lactamase genes (including (i.e. bla $a_{\mathrm{KPC}}$, bla $a_{\mathrm{NDM}}$, bla $a_{\mathrm{OXA}-48-\mathrm{like}}$, bla $_{\mathrm{IMP}}$ and $b l a_{\mathrm{VIM}}$ ) that takes about 6 hours to 
Table 3. Microarray-based methods for the detection of carbapenemase genes.

Target carbapenemase genes

\begin{tabular}{|c|c|c|c|c|c|}
\hline \multirow[b]{2}{*}{ Assay name } & \multicolumn{2}{|c|}{ KPC, NDM, OXA-48- } & \multirow[b]{2}{*}{ Other genes } & \multirow[b]{2}{*}{ Comment } & \multirow[b]{2}{*}{ Reference } \\
\hline & like, IMP, VIM & Others & & & \\
\hline $\begin{array}{l}\text { Check-MDR CT103 } \\
\text { (Checkpoints) }\end{array}$ & All & $\begin{array}{l}\text { GES, GIM, SPM, OXA-23, } \\
\text { OXA-24, OXA-58 }\end{array}$ & ESBLs, AmpCs & Run time: $6 \mathrm{~h}$ & {$[6]$} \\
\hline $\begin{array}{l}\text { Verigene }{ }^{\circledR} \text { BC-GN } \\
\text { (Nanoshpere, Illinois, USA) }\end{array}$ & All & $\begin{array}{l}\text { CTX-M group, OXA-23, } \\
\text { OXA-24, OXA-58 }\end{array}$ & $\begin{array}{l}\text { Genes for identification of } 9 \\
\text { gram-negative bacteria }\end{array}$ & $\begin{array}{l}\text { Proprietary, automated } \\
\text { system for blood culture. } \\
\text { Run time: } 2 \mathrm{~h} \text {. }\end{array}$ & [7] \\
\hline
\end{tabular}


complete the procedure [73] (Table 3). The major limitations are the high cost, turn-around time and labor associated with the procedure. Verigene ${ }^{\circledR}$ system (Nanoshere, Illinois, USA) is an automated microarray-based gene detection system and developed the Verigene ${ }^{\circledR}$ BC-GN test that has the ability to identify Gram negative bacteria and detects several genetic resistance determinants (including $b l a_{\mathrm{KPC}}, b l a_{\mathrm{NDM}}, b l a_{\mathrm{OXA}-48-\mathrm{like}}, b l a_{\mathrm{IMP}}, b l a_{\mathrm{VIM}}$ and $\left.b l a_{\mathrm{CTX}-\mathrm{M}}\right)$. This assay is expensive but can be performed directly on positive blood culture bottles with quick turn-around time of 2 hours [74].

Electrochemical DNA biosensor is a new device that detects hybridization of DNA to a biosensor component (usually complementary single-stranded DNA) by electrical transducer or optical detector. One recent report described application of this technology for the detection of a partial KPC gene [75].

\section{c. Next generation sequencing}

Next generation sequencing (NGS) is a new high-throughput DNA sequencing technology that can read large amounts of DNA sequences in a rapid fashion. After a launch of the first NGS instrument in 2005, this technology (also referred to as second generation sequencing) has continuously evolved to become more accurate, faster, easier to perform, and more cost effective. Recent NGS systems can determine the sequences of over 100 whole bacterial genomes during a single run. NGS systems provide sufficient data for the assessment of target genes and genetic relatedness between isolates. In fact, WGS has been used in the detailed analysis of CPE, especially 
during outbreak investigations [76] and large-scale surveillance [77].

Advantages of NGS for the detection of resistance genes include the following: 1) Predefined target genes are not required and novel elements can be discovered. 2) NGS provides comprehensive gene characteristics including antimicrobial resistance genes, clonal relatedness, plasmid replicon types, virulence genes, mobile genetic elements, and phage types. 3) NGS is becoming cost-effective and less time consuming than standard sequencing techniques.

The major disadvantage of WGS is data analysis. Bioinformatics and high-performance computing environment are required to process and analyze such a large amount of raw sequence data. Easy-to-use software packages or public web-based systems are available: for example, iMetAMOS (genome assembly and annotation pipeline; http://www.cbcb.umd.edu/software/imetamos), CLC genomics workbench (commercial NGS tools suite; CLC bio, Denmark), Galaxy (web-based NGS tools suite; https://galaxyproject.org/). After the assembly of a draft genome, several resistance gene databases such as ResFinder [78] and ARG-ANNOT [79], can be utilized for the discovery of resistance genes. ResFinder has a web-based interface and can accept raw sequence data before genome assembly has taken place. The ResFinder system was able to predict antimicrobial susceptibility with $99.7 \%$ concordance [77]. The ARG-ANNOT system includes a free sequence editing software named BioEdit. Both systems uses sequence similarity searching software such as BLAST [80]. 
The availability of cost effective NGS combined with more user friendly and rapid bioinformatics, has the potential to replace other molecular methods in the near future for the identification of carbapenemase genes.

\section{Expert commentary}

\section{Why and when should clinical laboratories test for carbapenemases?}

The emerging of resistance to the carbapenems among the Enterobacteriaceae is of special concern to the medical community at large since these agents are often the last line of effective therapy available for the treatment of infections caused by multidrug-resistant isolates [81]. Resistance to carbapenems involves multiple mechanisms, including alterations in outer membrane permeability mediated by the loss of porins, upregulation of efflux systems combined with high levels of AmpC cephalosporinases or other $\beta$-lactamases, however, the production of carbapenemases remains the most important mediators [1]. Carbapenemases are often part of mobile genetic elements such as plasmids that has the ability to easily move between different bacteria (i.e. horizontal transfer) (3). Controlling the spread of antibiotic resistance per say is a global public health problem.

The clinical laboratory acts as an early warning system, alerting the medical community to new resistance mechanisms present in clinically important bacteria. The presence of CPE in some instances, can be infection control emergencies and clinical laboratories should be able to rapidly 
detect carbapenemases among members of the Enterobacteriaceae; especially when these enzymes are first introduced into the local bacterial population [8]. CLSI and EUCAST do not recommend routine patient confirmation tests for carbapenemases if the current carbapenem interpretive criteria are used. This issue remains debatable since some observations had shown the importance of carbapenemases for the choice of therapeutic regimens [81]. However, the detection of CPE is a critical initial step required for appropriate management of patients during infection prevention and control efforts. Moreover, CPE testing is also appropriate for surveillance and epidemiological studies.

\section{How do methods perform for detecting different carbapenemases?}

Phenotypic methods show wide ranges in sensitivities and specificities for their abilities to detect different carbapenemases. As a general rule of thumb, the MHT is good for identifying CPEs with bla $a_{\mathrm{KPC}}$, and $b l a_{\mathrm{OXA}-48-\text { like }}$ enzymes [35]; Inhibitor-based tests are good for CPEs with bla $a_{\mathrm{KPC}}$, bla $_{\mathrm{NDM}}$ and when combined with temocillin are sufficient for those with $b l a_{\mathrm{OXA}-48-\text {-ike }}[32]$; CarbaNP test (and modifications) is excellent for CPEs with bla $a_{\mathrm{KPC}}, b l a_{\mathrm{NDM}}, b l a_{\mathrm{IMP}}$, and $b l a_{\mathrm{VIM}}[82]$ while the CIM test are good for CPEs with bla $a_{\mathrm{KPC}}$, bla $_{\mathrm{NDM}}$, bla $_{\mathrm{OXA}-48-\mathrm{like}}$, bla $_{\mathrm{IMP}}$, bla $_{\mathrm{VIM}}$ [38]. Please refer to Table 4 for details on the performance of phenotypic tests $[83,84]$.

Genotypic methods (including in-house and commercial tests) show the best sensitivities and specificities (as compared to phenotypic tests) for detecting different carbapenemase genes 
Table 4. Sensitivities of Phenotypic methods for the detection of Enterobacteriaceae with carbapenemases

\begin{tabular}{lcccc}
\hline Carbapenemase & Modified Hodge test & $\begin{array}{c}\text { Inhibitor based tests with } \\
\text { temocillin }\end{array}$ & CarbaNP & CIM \\
\hline KPC & $\mathbf{9 8 - 9 9 \%}$ & $\mathbf{9 3 - 9 5 \%}$ & $\mathbf{1 0 0 \%}$ & $\mathbf{1 0 0 \%}$ \\
NDM & $\mathbf{3 4 - 9 9 \%}$ & $\mathbf{9 0 - 9 5 \%}$ & $\mathbf{1 0 0 \%}$ & $\mathbf{1 0 0 \%}$ \\
VIM, IMP & $\mathbf{1 2 - 8 5 \%}$ & $\mathbf{8 5 - 9 0 \%}$ & $\mathbf{3 8 . 5 - 9 0 \%}$ & $\mathbf{9 6 - 1 0 0 \%}$ \\
OXA-48-like & $\mathbf{9 3 - 1 0 0 \%}$ & $100 \%$ & \\
\hline
\end{tabular}

References: [28], [31], [32], [34], [36], [37], [39], [40], [80] [83], [84] 
among CPEs. However, the ability of some commercial PCR assays to detect different carbapenemase genes significantly differ between assays [71]. Both phenotypic and same molecular methods have problems in the detection of OXA-48-like producer. Therefore, special attention should be given to the detection of OXA-48-like carbapenemases in endemic regions and should also be a priority in patients that had recently visit such an endemic region.

\section{A practical approach for the detection of carbapenemases}

We recommend that laboratory methods suitable for testing of CPE should at least be able to reliably detect the most clinical relevant carbapanemases (i.e. KPC, NDM, OXA-48-like, IMP and VIM) in a reasonably rapid fashion. We want to provide an approach for those clinical laboratories that do not have the necessary expertise for the detection of the most common types of CPEs. Our approach is practical and easy to introduce into the work flow of a clinical laboratory and will ensure that the most common types of CPEs are detected on a rapid fashion. It is important to remember that knowledge regarding the local epidemiology of carbapenemase types will always play an important role on deciding which laboratory methods are best suited to that specific region. We do acknowledge that rapid turnaround time is not required for CPE testing for surveillance and epidemiological surveys.

A simple approach for infection prevention and control efforts consists of two-steps namely a screening process (i.e. susceptibility testing with the carbapenems [i.e. ertapenem with meropenem, 
or imipenem using CLSI or EUCAST breakpoints]), followed by a confirmation test (i.e. phenotypic or genotypic) for the presence of a carbapenemase in isolates that test non-susceptibility to one or more of the carbapenems.

Phenotypic tests, in general terms, are simple to perform, interpret and can easily be introduced into the workflow of a clinical laboratory. For the rapid phenotype confirmation of CPE, the standardized Carba NP test as depicted in the CLSI 2015 guidelines [20] is most likely the best choice considering their performances in isolates with KPC and MBLs [82]. This rapid tests can easily be implemented in routine workflow of a clinical laboratory [43]. An inhibitor-based approach combined with temocillin susceptibility, the CIM or the MHT with an easy-to-follow algorithms, also performs adequately [32]. However, these assays are time consuming and add another 18-24 hours to the reporting of the final result.

PCR-based molecular confirmation methods (in-house and commercial assays) have excellent sensitivities and specificities but are unfortunately rather expensive and time consuming (as compared to most phenotypic tests). Figure 1 provides an easy-to-follow algorithm for the detection of carbapenemase among Enterobacteriaceae.

\section{Five-year review}

Rapid phenotypic methods, most likely the Carba NP-based tests, will be used as routine testing in most clinical laboratories. The developing countries, few clinical laboratories will 


\section{Figure 1. Detection of carbapenemases in Enterobacteriaceae}

\section{Enterobacteriaceae}

Screen positive: non-susceptible to ERT or MEM or IMI

(CLSI or EUCAST breakpoints)

Rapid hydrolysis tes

(Carba NP test or modifications)

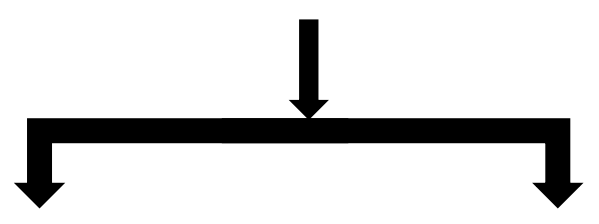

\begin{tabular}{c|c|}
\hline $\begin{array}{c}\text { Positive } \\
\text { Report as carbapenemase producer }\end{array}$ & $\begin{array}{c}\text { Negative } \\
\text { Report as additional tests to follow } \\
\text { Perform PCR-based test or refer to } \\
\text { reference laboratory } \\
\text { (e.g. different types), perform PCR- } \\
\text { based test or refer to reference } \\
\text { laboratory. } \\
\text { Consider doing MHT, CIM or inhibitor } \\
\text { based tests with temocillin if PCR not } \\
\text { readily available }\end{array}$ \\
$\begin{array}{c}\text { Consider doing inhibitor based tests } \\
\text { with temocillin if PCR not readily } \\
\text { available. }\end{array}$ \\
\hline $\begin{array}{c}\text { If PCR, MHT, CIM or inhibitor based tests positive: } \\
\text { report as as carbapenemase producer } \\
\text { If PCR, MHT, CIM or inhibitor based tests } \\
\text { negative: report as non carbapenemase producer }\end{array}$ \\
\hline
\end{tabular}


implement automated PCR-based detection methods. Such convenient molecular methods will also be used for the direct detection of CPE from clinical specimen, which will shorten current turn-around time (usually several days to hours) and impact infection control measures of patients with CPE. However, the nightmare of CPE spread will continue across hospitals, healthcare-associated facilities, and countries, despite implementation of infection control prevention measures $[85,86]$.

The antibiotic pressure in healthcare settings and other environments will continue select for variants of carbapenemases and CPE with these enzymes will continue to spread globally. The strong need to control CPE will further promote research on development and application of diagnostic technologies. NGS and MALDI-TOF applications are the most promising methods and will advance further for the detection of CPEs.

\section{Key issues}

1. CPE is an emerging global public health threat that can easily spread among patients.

2. Carbapenemases are versatile $\beta$-lactamases, which complicates their detection. The five clinical relevant carbapenemases are KPC, NDM, OXA-48-like, IMP, and VIM enzymes.

3. CLSI and EUCAST do not recommend routine patient confirmation tests for carbapenemases if the current carbapenem interpretive criteria are used.

4. The detection of $\mathrm{CPE}$ is a critical initial step required for appropriate management of patients 
during infection prevention and control efforts. Moreover, CPE testing is also appropriate for surveillance and epidemiological studies.

5. Screening for non-susceptibility to the carbapenems is the important initial step to successfully detect CPE and should be followed by phenotypic or genotypic confirmation tests.

6. For the rapid phenotype confirmation of CPE, the standardized CLSI version of Carba NP test or the use of commercial versions is most likely the best choice for laboratories that have limited access to molecular methods.

7. An inhibitor-based approach combined with temocillin susceptibility, the carbapenem inactivation method or the MHT with an easy-to-follow algorithms, also performs adequately.

8. The molecular procedures to detect CPE have the best sensitivities and specificities. Multiplex real-time PCR assays allow reliable detection in most clinical laboratories.

9. The detection of certain carbapenemases (e.g. OXA-48-like) with phenotypic methods can be challenging due to their weak carbapenemase activities.

10. Recent advances in NGS and MALDI-TOF MS applications are promising for the detection of CPE.

\section{Funding}

This work was supported in part by a research grant from Calgary Laboratory Services (\#10009392) and Kyoto University Graduate School of Medicine (\#10012050) 


\section{References}

1. Paterson DL. Resistance in gram־negative bacteria: Enterobacteriaceae. Am J Infect Control, 34(5 Suppl 1), S20-28; discussion S64-73 (2006).

2. America IDSo. The $10 \mathrm{x}$ '20 Initiative: pursuing a global commitment to develop 10 new antibacterial drugs by 2020. Clin Infect Dis, 50(8), 1081-1083 (2010).

3. World Health Organization. Antimicrobial resistance: global report on surveillance 2014 . (Ed.^(Eds) (2014)

4. Schwaber MJ, Navon-Venezia S, Kaye KS, Ben-Ami R, Schwartz D, Carmeli Y. Clinical and economic impact of bacteremia with extended- spectrum-beta-lactamase-producing Enterobacteriaceae. Antimicrob Agents Chemother, 50(4), 1257-1262 (2006).

5. Nordmann P, Dortet L, Poirel L. Carbapenem resistance in Enterobacteriaceae: here is the storm! Trends in molecular medicine, 18(5), 263-272 (2012).

6. Pitout JD, Nordmann P, Poirel L. Carbapenemase-Producing Klebsiella pneumoniae, a Key Pathogen Set for Global Nosocomial Dominance. Antimicrobial agents and chemotherapy, 59(10), 5873-5884 (2015).

7. Nordmann P, Naas T, Poirel L. Global spread of Carbapenemase-producing Enterobacteriaceae. Emerging infectious diseases, 17(10), 1791-1798 (2011).

8. Nordmann P, Poirel L. The difficult-to-control spread of carbapenemase producers among Enterobacteriaceae worldwide. Clinical microbiology and infection : the official publication of the European Society of Clinical Microbiology and Infectious Diseases, 20(9), 821-830 (2014). 
9. Ahmed-Bentley J, Chandran AU, Joffe AM, French D, Peirano G, Pitout JD. Gram-negative bacteria that produce carbapenemases causing death attributed to recent foreign hospitalization. Antimicrob Agents Chemother, 57(7), 3085-3091 (2013).

10. Munoz-Price LS, Poirel L, Bonomo RA et al. Clinical epidemiology of the global expansion of Klebsiella pneumoniae carbapenemases. The Lancet. Infectious diseases, 13(9), 785-796 (2013).

11. Walther-Rasmussen J, Hoiby N. Class A carbapenemases. J Antimicrob Chemother, 60(3), 470-482 (2007).

12. Queenan AM, Bush K. Carbapenemases: the versatile beta-lactamases. Clinical microbiology reviews, 20(3), 440-458 (2007).

13. Deshpande LM, Rhomberg PR, Sader HS, Jones RN. Emergence of serine carbapenemases (KPC and SME) among clinical strains of Enterobacteriaceae isolated in the United States Medical Centers: report from the MYSTIC Program (1999-2005). Diagn Microbiol Infect Dis, 56(4), 367-372 (2006).

14. Nordmann P, Cuzon G, Naas T. The real threat of Klebsiella pneumoniae carbapenemase-producing bacteria. The Lancet. Infectious diseases, 9(4), 228-236 (2009).

15. Poirel L, Potron A, Nordmann P. OXA-48-like carbapenemases: the phantom menace. The Journal of antimicrobial chemotherapy, 67(7), 1597-1606 (2012).

16. Poirel L, Heritier C, Spicq C, Nordmann P. In vivo acquisition of high-level resistance to imipenem in Escherichia coli. Journal of clinical microbiology, 42(8), 3831-3833 (2004). 
17. Oueslati S, Nordmann P, Poirel L. Heterogeneous hydrolytic features for OXA-48-like beta-lactamases. The Journal of antimicrobial chemotherapy, 70(4), 1059-1063 (2015).

18. Nordmann P, Gniadkowski M, Giske CG et al. Identification and screening of carbapenemase-producing Enterobacteriaceae. Clin Microbiol Infect, 18(5), 432-438 (2012).

19. Huang TD, Poirel L, Bogaerts P, Berhin C, Nordmann P, Glupczynski Y. Temocillin and piperacillin/tazobactam resistance by disc diffusion as antimicrobial surrogate markers for the detection of carbapenemase-producing Enterobacteriaceae in geographical areas with a high prevalence of OXA-48 producers. The Journal of antimicrobial chemotherapy, 69(2), 445-450 (2014).

20. CLSI. Performance standards for antimicrobial susceptibility testing; Twenty-fifth informational supplement. CLSI document M100-S25. Wayne, PA: Clinical and Laboratory Standards Institute. $\left(\mathrm{Ed} .^{\wedge}(\mathrm{Eds})(2015)\right.$

21. EUCAST subcommittee for detection of resistance mechanisms and specific resistances of clinical and/or epidemiological importance. EUCAST guidelines for detection of resistance mechanisms and specific resistances of clinical and/or epidemiological importance, Version 1.0. Available from http://www.eucast.org/fileadmin/src/media/PDFs/EUCAST_files/Resistance_mechanisms/EUCAS T_detection_of_resistance_mechanisms_v1.0_20131211.pdf. (Ed.^(Eds) (2013)

22. Day KM, Pike R, Winstanley TG et al. Use of faropenem as an indicator of carbapenemase activity in the Enterobacteriaceae. J Clin Microbiol, 51(6), 1881-1886 (2013).

23. Lee M, Chung HS. Different antimicrobial susceptibility testing methods to detect ertapenem resistance in Enterobacteriaceae: VITEK2, MicroScan, Etest, disk diffusion, and broth 
microdilution. J Microbiol Methods, 112, 87-91 (2015).

24. Doi Y, Paterson DL. Carbapenemase-producing Enterobacteriaceae. Semin Respir Crit Care Med, 36(1), 74-84 (2015).

25. Doi Y, Potoski BA, Adams-Haduch JM, Sidjabat HE, Pasculle AW, Paterson DL. Simple disk-based method for detection of Klebsiella pneumoniae carbapenemase-type beta-lactamase by use of a boronic acid compound. Journal of clinical microbiology, 46(12), 4083-4086 (2008).

26. Giske CG, Gezelius L, Samuelsen O, Warner M, Sundsfjord A, Woodford N. A sensitive and specific phenotypic assay for detection of metallo-beta-lactamases and KPC in Klebsiella pneumoniae with the use of meropenem disks supplemented with aminophenylboronic acid, dipicolinic acid and cloxacillin. Clinical microbiology and infection : the official publication of the European Society of Clinical Microbiology and Infectious Diseases, 17(4), 552-556 (2011).

27. Tsakris A, Kristo I, Poulou A et al. Evaluation of boronic acid disk tests for differentiating KPC-possessing Klebsiella pneumoniae isolates in the clinical laboratory. Journal of clinical microbiology, 47(2), 362-367 (2009).

28. Doyle D, Peirano G, Lascols C, Lloyd T, Church DL, Pitout JD. Laboratory detection of Enterobacteriaceae that produce carbapenemases. J Clin Microbiol, 50(12), 3877-3880 (2012).

29. Miriagou V, Tzelepi E, Kotsakis SD, Daikos GL, Bou Casals J, Tzouvelekis LS. Combined disc methods for the detection of KPC- and/or VIM-positive Klebsiella pneumoniae: improving reliability for the double carbapenemase producers. Clin Microbiol Infect, 19(9), E412-415 (2013).

30. Tsakris A, Poulou A, Pournaras S et al. A simple phenotypic method for the differentiation of 
metallo-beta-lactamases and class A KPC carbapenemases in Enterobacteriaceae clinical isolates. The Journal of antimicrobial chemotherapy, 65(8), 1664-1671 (2010).

31. van Dijk K, Voets GM, Scharringa J et al. A disc diffusion assay for detection of class A, B and OXA-48 carbapenemases in Enterobacteriaceae using phenyl boronic acid, dipicolinic acid and temocillin. Clinical microbiology and infection : the official publication of the European Society of Clinical Microbiology and Infectious Diseases, 20(4), 345-349 (2014).

32. Maurer FP, Castelberg C, Quiblier C, Bloemberg GV, Hombach M. Evaluation of carbapenemase screening and confirmation tests with Enterobacteriaceae and development of a practical diagnostic algorithm. J Clin Microbiol, 53(1), 95-104 (2015).

33. Tsakris A, Poulou A, Bogaerts P, Dimitroulia E, Pournaras S, Glupczynski Y. Evaluation of a new phenotypic OXA-48 disk test for differentiation of OXA-48 carbapenemase-producing Enterobacteriaceae clinical isolates. Journal of clinical microbiology, 53(4), 1245-1251 (2015).

34. Carvalhaes CG, Picao RC, Nicoletti AG, Xavier DE, Gales AC. Cloverleaf test (modified Hodge test) for detecting carbapenemase production in Klebsiella pneumoniae: be aware of false positive results. The Journal of antimicrobial chemotherapy, 65(2), 249-251 (2010).

35. Girlich D, Poirel L, Nordmann P. Value of the modified Hodge test for detection of emerging carbapenemases in Enterobacteriaceae. Journal of clinical microbiology, 50(2), 477-479 (2012).

36. Mathers AJ, Carroll J, Sifri CD, Hazen KC. Modified Hodge test versus indirect carbapenemase test: prospective evaluation of a phenotypic assay for detection of Klebsiella pneumoniae carbapenemase (KPC) in Enterobacteriaceae. Journal of clinical microbiology, 51(4), 1291-1293 (2013). 
37. van der Zwaluw K, de Haan A, Pluister GN, Bootsma HJ, de Neeling AJ, Schouls LM. The carbapenem inactivation method (CIM), a simple and low-cost alternative for the Carba NP test to assess phenotypic carbapenemase activity in gram-negative rods. PLoS One, 10(3), e0123690 (2015)

38. Tijet N, Patel SN, Melano RG. Detection of carbapenemase activity in Enterobacteriaceae: comparison of the carbapenem inactivation method versus the Carba NP test. The Journal of antimicrobial chemotherapy, 71(1), 274-276 (2016).

39. Nordmann P, Poirel L, Dortet L. Rapid detection of carbapenemase-producing Enterobacteriaceae. Emerg Infect Dis, 18(9), 1503-1507 (2012).

40. Tijet N, Boyd D, Patel SN, Mulvey MR, Melano RG. Evaluation of the Carba NP test for rapid detection of carbapenemase-producing Enterobacteriaceae and Pseudomonas aeruginosa. Antimicrob Agents Chemother, 57(9), 4578-4580 (2013).

41. Vasoo S, Cunningham SA, Kohner PC et al. Comparison of a novel, rapid chromogenic biochemical assay, the Carba NP test, with the modified Hodge test for detection of carbapenemase-producing Gram-negative bacilli. J Clin Microbiol, 51(9), 3097-3101 (2013).

42. AbdelGhani S, Thomson GK, Snyder JW, Thomson KS. Comparison of the Carba NP, Modified Carba NP, and Updated Rosco Neo-Rapid CARB Kit Tests for Carbapenemase Detection. J Clin Microbiol, (2015).

43. Dortet L, Agathine A, Naas T, Cuzon G, Poirel L, Nordmann P. Evaluation of the RAPIDEC® CARBA NP, the Rapid CARB Screen ${ }^{\circledR}$ and the Carba NP test for biochemical detection of carbapenemase-producing Enterobacteriaceae. JAntimicrob Chemother, (2015). 
44. Dortet L, Poirel L, Nordmann P. Rapid identification of carbapenemase types in Enterobacteriaceae and Pseudomonas spp. by using a biochemical test. Antimicrob Agents Chemother, 56(12), 6437-6440 (2012).

45. Lee LY, Korman TM, Graham M. Rapid time to results and high sensitivity of the CarbaNP test on early cultures. J Clin Microbiol, 52(11), 4023-4026 (2014).

46. Dortet L, Bréchard L, Poirel L, Nordmann P. Rapid detection of carbapenemase-producing Enterobacteriaceae from blood cultures. Clin Microbiol Infect, 20(4), 340-344 (2014).

47. Kabir MH, Meunier D, Hopkins KL, Giske CG, Woodford N. A two-centre evaluation of RAPIDEC(R) CARBA NP for carbapenemase detection in Enterobacteriaceae, Pseudomonas aeruginosa and Acinetobacter spp. The Journal of antimicrobial chemotherapy, (2016).

48. Pires J, Novais A, Peixe L. Blue-carba, an easy biochemical test for detection of diverse carbapenemase producers directly from bacterial cultures. J Clin Microbiol, 51(12), 4281-4283 (2013).

49. Garcia-Fernandez S, Morosini MI, Gijon D et al. Detection of Carbapenemase Production in a Collection of Enterobacteriaceae with Characterized Resistance Mechanisms from Clinical and Environmental Origins by Use of Both Carba NP and Blue-Carba Tests. Journal of clinical microbiology, 54(2), 464-466 (2016).

50. Pasteran F, Tijet N, Melano RG, Corso A. Simplified Protocol for Carba NP Test for Enhanced Detection of Carbapenemase Producers Directly from Bacterial Cultures. Journal of clinical microbiology, 53(12), 3908-3911 (2015). 
51. Pires J, Tinguely R, Thomas B, Luzzaro F, Endimiani A. Comparison of the in-house made Carba-NP and Blue-Carba tests: Considerations for better detection of carbapenemase-producing Enterobacteriaceae. J Microbiol Methods, 122, 33-37 (2016).

52. Novais Â, Brilhante M, Pires J, Peixe L. Evaluation of the Recently Launched Rapid Carb Blue Kit for Detection of Carbapenemase-Producing Gram-Negative Bacteria. J Clin Microbiol, 53(9), 3105-3107 (2015).

53. Martino MD, Koga PC, Pasternak J et al. Evaluation of a new rapid test for carbapenemase detection in carbapenem resistant Enterobacteriaceae. J Microbiol Methods, 115, 20-21 (2015).

54. Notake S, Matsuda M, Tamai K, Yanagisawa H, Hiramatsu K, Kikuchi K. Detection of IMP metallo-B-lactamase in carbapenem-nonsusceptible Enterobacteriaceae and non-glucose-fermenting Gram-negative rods by immunochromatography assay. J Clin Microbiol, 51(6), 1762-1768 (2013).

55. Evrard S. [Rethinking clinical research in surgical oncology. From comic opera to quality control]. Bull Cancer, 103(1), 87-95 (2016).

56. Bogaerts P, Yunus S, Massart M, Huang TD, Glupczynski Y. Evaluation of the BYG Carba Test, a New Electrochemical Assay for Rapid Laboratory Detection of Carbapenemase-Producing Enterobacteriaceae. Journal of clinical microbiology, 54(2), 349-358 (2016).

57. Bernabeu S, Poirel L, Nordmann P. Spectrophotometry-based detection of carbapenemase producers among Enterobacteriaceae. Diagn Microbiol Infect Dis, 74(1), 88-90 (2012).

58. Dortet L, Bréchard L, Cuzon G, Poirel L, Nordmann P. Strategy for rapid detection of 
carbapenemase-producing Enterobacteriaceae. Antimicrob Agents Chemother, 58(4), 2441-2445 (2014).

59. Hrabák J, Studentová V, Walková R et al. Detection of NDM-1, VIM-1, KPC, OXA-48, and OXA-162 carbapenemases by matrix-assisted laser desorption ionization-time of flight mass spectrometry. J Clin Microbiol, 50(7), 2441-2443 (2012).

60. Burckhardt I, Zimmermann S. Using matrix-assisted laser desorption ionization-time of flight mass spectrometry to detect carbapenem resistance within 1 to 2.5 hours. $J$ Clin Microbiol, 49(9), 3321-3324 (2011).

61. Hrabák J, Chudáčková E, Papagiannitsis CC. Detection of carbapenemases in Enterobacteriaceae: a challenge for diagnostic microbiological laboratories. Clin Microbiol Infect, 20(9), 839-853 (2014).

62. Sauget M, Cabrolier N, Manzoni M, Bertrand X, Hocquet D. Rapid, sensitive and specific detection of OXA-48-like-producing Enterobacteriaceae by matrix-assisted laser desorption/ionization time-of-flight mass spectrometry. J Microbiol Methods, 105, 88-91 (2014).

63. Studentova V, Papagiannitsis CC, Izdebski $\mathrm{R}$ et al. Detection of OXA-48-type carbapenemase-producing Enterobacteriaceae in diagnostic laboratories can be enhanced by addition of bicarbonates to cultivation media or reaction buffers. Folia Microbiol (Praha), 60(2), 119-129 (2015).

64. Wang L, Han C, Sui W, Wang M, Lu X. MALDI-TOF MS applied to indirect carbapenemase detection: a validated procedure to clearly distinguish between carbapenemase-positive and carbapenemase-negative bacterial strains. Anal Bioanal Chem, 405(15), 5259-5266 (2013). 
65. Papagiannitsis CC, Študentová V, Izdebski $\mathrm{R}$ et al. Matrix-assisted laser desorption ionization-time of flight mass spectrometry meropenem hydrolysis assay with $\mathrm{NH} 4 \mathrm{HCO} 3$, a reliable tool for direct detection of carbapenemase activity. $J$ Clin Microbiol, 53(5), 1731-1735 (2015).

66. Lau AF, Wang H, Weingarten RA et al. A rapid matrix-assisted laser desorption ionization-time of flight mass spectrometry-based method for single-plasmid tracking in an outbreak of carbapenem-resistant Enterobacteriaceae. J Clin Microbiol, 52(8), 2804-2812 (2014).

67. Youn JH, Drake SK, Weingarten RA, Frank KM, Dekker JP, Lau AF. Clinical Performance of a Matrix-Assisted Laser Desorption Ionization Time-of-Flight Mass Spectrometry Method for the Detection of Certain blaKPC-containing Plasmids. J Clin Microbiol, (2015).

68. Poirel L, Walsh TR, Cuvillier V, Nordmann P. Multiplex PCR for detection of acquired carbapenemase genes. Diagn Microbiol Infect Dis, 70(1), 119-123 (2011).

69. Monteiro J, Widen RH, Pignatari AC, Kubasek C, Silbert S. Rapid detection of carbapenemase genes by multiplex real-time PCR. JAntimicrob Chemother, 67(4), 906-909 (2012).

70. Lee TD, Adie K, McNabb A et al. Rapid Detection of KPC, NDM, and OXA-48-Like Carbapenemases by Real-Time PCR from Rectal Swab Surveillance Samples. J Clin Microbiol, 53(8), 2731-2733 (2015).

71. Findlay J, Hopkins KL, Meunier D, Woodford N. Evaluation of three commercial assays for rapid detection of genes encoding clinically relevant carbapenemases in cultured bacteria. J Antimicrob Chemother, 70(5), 1338-1342 (2015). 
72. García-Fernández S, Morosini MI, Marco F et al. Evaluation of the eazyplex® SuperBug CRE system for rapid detection of carbapenemases and ESBLs in clinical Enterobacteriaceae isolates recovered at two Spanish hospitals. J Antimicrob Chemother, 70(4), 1047-1050 (2015).

73. Braun SD, Monecke S, Thürmer A et al. Rapid identification of carbapenemase genes in gram-negative bacteria with an oligonucleotide microarray-based assay. PLoS One, 9(7), e102232 (2014).

74. Tojo M, Fujita T, Ainoda Y et al. Evaluation of an automated rapid diagnostic assay for detection of Gram-negative bacteria and their drug-resistance genes in positive blood cultures. PLoS One, 9(4), e94064 (2014).

75. Pan HZ, Yu HW, Wang N et al. Electrochemical DNA biosensor based on a glassy carbon electrode modified with gold nanoparticles and graphene for sensitive determination of Klebsiella pneumoniae carbapenemase. J Biotechnol, (2015).

76. Reuter S, Ellington MJ, Cartwright EJ et al. Rapid bacterial whole-genome sequencing to enhance diagnostic and public health microbiology. JAMA Intern Med, 173(15), 1397-1404 (2013).

77. Zankari E, Hasman H, Kaas RS et al. Genotyping using whole-genome sequencing is a realistic alternative to surveillance based on phenotypic antimicrobial susceptibility testing. J Antimicrob Chemother, 68(4), 771-777 (2013).

78. Zankari E, Hasman $\mathrm{H}$, Cosentino $\mathrm{S}$ et al. Identification of acquired antimicrobial resistance genes. JAntimicrob Chemother, 67(11), 2640-2644 (2012).

79. Gupta SK, Padmanabhan BR, Diene SM et al. ARG-ANNOT, a new bioinformatic tool to discover 
antibiotic resistance genes in bacterial genomes. Antimicrob Agents Chemother, 58(1), 212-220 (2014).

80. Camacho C, Coulouris G, Avagyan V et al. BLAST+: architecture and applications. BMC Bioinformatics, 10, 421 (2009).

81. Tzouvelekis LS, Markogiannakis A, Psichogiou M, Tassios PT, Daikos GL. Carbapenemases in Klebsiella pneumoniae and other Enterobacteriaceae: an evolving crisis of global dimensions. Clinical microbiology reviews, 25(4), 682-707 (2012).

82. Osterblad M, Hakanen AJ, Jalava J. Evaluation of the Carba NP test for carbapenemase detection. Antimicrobial agents and chemotherapy, 58(12), 7553-7556 (2014).

83. Huang TD, Berhin C, Bogaerts P, Glupczynski Y. Comparative evaluation of two chromogenic tests for rapid detection of carbapenemase in Enterobacteriaceae and in Pseudomonas aeruginosa isolates. Journal of clinical microbiology, 52(8), 3060-3063 (2014).

84. Kim HK, Park JS, Sung H, Kim MN. Further Modification of the Modified Hodge Test for Detecting Metallo-beta-Lactamase-Producing Carbapenem-Resistant Enterobacteriaceae. Ann Lab Med, 35(3), 298-305 (2015).

85. Akova M, Daikos GL, Tzouvelekis L, Carmeli Y. Interventional strategies and current clinical experience with carbapenemase-producing Gram-negative bacteria. Clin Microbiol Infect, 18(5), 439-448 (2012).

86. Kuehn BM. "Nightmare" bacteria on the rise in US hospitals, long-term care facilities. JAMA, 309(15), 1573-1574 (2013). 


\section{Highlighted References}

[28] $114 \mathrm{CPE}$ including all of the five major carbapenemases and 28 non-CPE clinical isolates were used to compare detection performance of MHT and two commercial inhibitor-based disk combination kits. One-reaction multiplex PCR assay was also described.

[77] ResFinder, web-based comprehensive detection system of all the known resistance genes using WGS data.

[39] The first paper to describe the Carba NP test for the detection of carbapenemase. The original concept, protocol and interpretation (color change) are described.

[58] Detection performance of the Carba NP test, UV spectrophotometry, and a commercial DNA microarray were compared prospectively for 862 Enterobacteriaceae isolates. The authors also proposed a detection algorithm.

[59] MALDI-TOF MS-based detection of meropenem degradation product. Its concept and changes in spectra are shown. 